\title{
THE CRYSTAL STRUCTURE OF ASPIRIN ACETYLATED HUMAN CYCLOOXYGENASE-2: INSIGHT INTO THE FORMATION OF PRODUCTS WITH REVERSED STEREOCHEMISTRY
}

\author{
Michael J. Lucido, Benjamin J. Orlando, Alex J. Vecchio ${ }^{\dagger}$, and Michael G. Malkowski ${ }^{\star}$ \\ Department of Structural Biology, The State University of New York at Buffalo and Hauptman- \\ Woodward Medical Research Institute, Buffalo, NY 14203, USA
}

\begin{abstract}
Aspirin and other nonsterroidal anti-inflammatory drugs target the Cyclooxygenase enzymes (COX-1 and COX-2) to block the formation of prostaglandins. Aspirin is unique in that it covalently modifies each enzyme by acetylating Ser-530 within the cyclooxygenase active site. Acetylation of COX-1 leads to complete loss of activity, while acetylation of COX-2 results in the generation of the mono-oxygenated product 15(R)-hydroxyeicosatetraenoic acid (15R-HETE). Ser-530 has also been shown to influence the stereochemistry for oxygen addition into the prostaglandin product. We determined the crystal structures of S530T murine (mu) COX-2, aspirin-acetylated human (hu) COX-2, and huCOX-2 in complex with salicylate to $1.9 \AA 2.0 \AA$, and $2.4 \AA$, respectively. The structures reveal that: 1 ) the acetylated Ser-530 completely blocks access to the hydrophobic groove; 2 ) the observed binding pose of salicylate is reflective of the enzyme-inhibitor complex prior to acetylation; and 3) the observed Thr-530 rotamer in the S530T muCOX-2 crystal structure does not impede access to the hydrophobic groove. Based on these structural observations, along with functional analysis of the S530T/G533V double mutant, we propose a working hypothesis for the generation of 15R-HETE by aspirin-acetylated COX-2. We also observe differential acetylation of COX-2 purified in various detergent systems and nanodiscs, indicating that detergent and lipid binding within the membrane-binding domain of the enzyme alters the rate of the acetylation reaction in vitro.
\end{abstract}

Cyclooxygenase enzymes (COX-1 and COX-2) are bifunctional homodimeric enzymes that oxygenate arachidonic acid (AA) to generate prostaglandin $\mathrm{H}_{2}\left(\mathrm{PGH}_{2}\right)$ in the committed step of prostaglandin (PG) biosynthesis ${ }^{1}$. $\mathrm{PGH}_{2}$ serves as the major substrate for downstream synthases that give rise to this potent class of signaling molecules, which are responsible for the control of physiological "housekeeping" functions, including the regulation of renal water and sodium metabolism, parturition, and stomach acid secretion ${ }^{2}$. Abnormal changes in PG production are associated with various disease pathologies,

\footnotetext{
*Address correspondence to: Michael G. Malkowski, Department of Structural Biology, The State University of New York at Buffalo, 700 Ellicott Street, Buffalo, New York 14203. Tel: (716) 898-8624; Fax: (716) 898-8660; mgm22@ buffalo.edu.

${ }^{\dagger}$ Current Address: Department of Biochemistry and Biophysics, University of California, San Francisco, San Francisco, CA 94158 SUPPORTING INFORMATION

Supporting Information is available and may be accessed free of charge online at http://pubs.acs.org.

Supplementary tables and figure defining the interactions made by acetylated Ser-530 and salicylate with residues lining the cyclooxygenase channel.
} 
including inflammation, cardiovascular disease, and cancer ${ }^{3}$. Aspirin, ibuprofen, and other nonselective nonsterroidal anti-inflammatory drugs (NSAIDs) inhibit the synthesis of $\mathrm{PGH}_{2}$ by COX-1 and COX-2, resulting in the reduction of acute and chronic inflammation and in the case of aspirin, protection against adverse cardiovascular events ${ }^{3,4}$. COX-2 is also selectively inhibited in a time-dependent manner by diaryl-heterocycle-based compounds, including rofecoxib and celecoxib ${ }^{5}$.

Each monomer of COX contains a separate cyclooxygenase and peroxidase active site, which are functionally linked by a bridging heme moiety. For the production of $\mathrm{PGH}_{2}, \mathrm{AA}$ binds within the cyclooxygenase channel with its carboxylate moiety located near Arg-120 and Tyr-355 at the channel entrance and with its $\omega$-end located in a hydrophobic groove above the side chain of Ser-530 where it abuts Gly-533 ${ }^{6,7}$. In this pose, carbon-13 is placed below the phenolic oxygen of Tyr-385. A preliminary catalytic turnover is required at the peroxidase active site to generate an oxy-ferryl porphyrin cation radical, which is then transferred to Tyr-385 to initiate the cyclooxygenase reaction ${ }^{8}$. Following abstraction of the 13pro-S hydrogen from AA by the tyrosyl radical, two molecules of oxygen are added to produce the intermediate prostaglandin $\mathrm{G}_{2}\left(\mathrm{PGG}_{2}\right)$. $\mathrm{PGG}_{2}$ is then released to the peroxidase active site, where the 15-hydroperoxide group of $\mathrm{PGG}_{2}$ is reduced to form $\mathrm{PGH}_{2}$. In addition to $\mathrm{PGH}_{2}$, small amounts of the monohydroxy acids 11(R)-hydroxyeicosatetraenoic acid (11R-HETE) and 15(S)-hydroxyeicosatetraenoic acid (15S-HETE) are generated as byproducts of the cyclooxygenase reaction ${ }^{9}$.

COX enzymes are homodimers consisting of tightly associated monomers that dissociate only upon denaturation ${ }^{10}$. Recent studies have shown that only one monomer of the COX homodimer is active at a given time ${ }^{11}$. The monomers act additionally through an allosteric/ catalytic couple, with AA oxygenation being modulated in the "catalytic" monomer $\left(\mathrm{E}_{\mathrm{cat}}\right)$ through the binding of dietary nonsubstrate fatty acids (nsFAs) and nonselective NSAIDs to the opposite monomer, the "allosteric" monomer $\left(\mathrm{E}_{\text {allo }}\right){ }^{12-16}$. The mechanism responsible for governing inter-monomer communication is unknown. To date, $\mathrm{X}$-ray crystal structures of COX have not provided insight into the conformational motions responsible for communication between $\mathrm{E}_{\text {allo }}$ and $\mathrm{E}_{\mathrm{cat}}$.

Aspirin is unique amongst the NSAIDs in that it covalently modifies COX-1 and COX-2 in a time-dependent manner via the acetylation of the hydroxyl group of Ser-530 ${ }^{17,18}$. The acetylation of Ser-530 by aspirin has differential effects on the activity and product profiles of COX-1 and COX-2. Aspirin treatment of COX-1 irreversibly inhibits the cyclooxygenase activity of the enzyme and subsequently the production of $\mathrm{PGG}_{2}$. Analysis of the crystal structure of ovine (ov) COX-1 acetylated by 2-bromoacetoxy-benzoic acid suggests that the productive binding of AA within the cyclooxygenase channel would be blocked upon acetylation of Ser-530 ${ }^{19}$. Conversely, aspirin acetylation of COX-2 results in a shift in reaction specificity, converting enzyme activity from a cyclooxygenase to a lipoxygenase, resulting in the generation of 15R-HETE ${ }^{17,20}$. The 15R-HETE generated from aspirinacetylated COX-2 also exhibits a reversal of the R/S stereochemistry for oxygen at carbon- 15 compared to the products generated by the uninhibited enzyme. Although COX-1 and COX-2 are sequence homodimers, recent studies suggest that only one monomer is acetylated upon treatment with aspirin 21,22 . 
There are mechanistic and structural aspects related to the generation of 15R-HETE by aspirin-acetylated COX-2 yet to be resolved ${ }^{23}$. These include how the stereochemistry for oxygen addition at carbon- 15 is controlled and the nature of the conformation of AA within the acetylated cyclooxygenase channel that leads to the production of 15R-HETE. Numerous groups have proposed that AA binds in an unconventional or bent conformation, compared to the productive conformation leading to $\mathrm{PGG}_{2}$ formation, to facilitate the stereospecific addition of oxygen at carbon-15 ${ }^{24-26}$. However, mutagenesis studies suggest that AA binds to wild type and acetylated COX-2 utilizing a similar pose ${ }^{27}$. It has also been proposed that the acetylated Ser-530 residue causes a strain on the geometry of the developing pentadienyl radical of AA, resulting in the trapping of the radical on carbon-15 for subsequent oxygen addition ${ }^{28}$. Although proposed separately, these models are not mutually exclusive, with the potential for some combination responsible for the genesis of 15R-HETE by aspirin-acetylated COX-2 23 .

Here we report structure-function studies designed to provide additional insight into the production of 15R-HETE by aspirin-acetylated human (hu) COX-2. We determined the Xray crystal structures of aspirin-acetylated huCOX-2 and huCOX-2 in complex with the aspirin precursor salicylic acid to $2.04 \AA$ and $2.38 \AA$ resolution, respectively. The structures provide the first experimental observations of the molecular interactions made by the acetylated Ser-530 side chain and salicylic acid in the cyclooxygenase channel of huCOX-2. Through the combination of structural and functional observations presented here and published previously, we put forth a working hypothesis of how AA binds within the acetylated COX-2 cyclooxygenase channel to generate 15R-HETE.

\section{EXPERIMENTAL PROCEDURES}

\section{Materials}

Arachidonic acid (AA; 5Z, 8Z, 11Z, 14Z-eicosatetraenoic acid), ibuprofen, flurbiprofen, and acetylsalicylic acid were purchased from Cayman Chemical Company (Ann Arbor, MI). Celebrex ${ }^{\circledR}$ (celecoxib) was from a physician sample. $\left[1-{ }^{14} \mathrm{C}\right] \mathrm{AA}(55 \mathrm{mCi} / \mathrm{mmol})$ and [acetyl- ${ }^{14} \mathrm{C}$ ] ASA $(55 \mathrm{mCi} / \mathrm{mmol})$ were purchased from American Radiolabeled Chemicals (St. Louis, MO). $\mathrm{Fe}^{3+}$-protoporphyrin IX and $\mathrm{Co}^{3+}$-protoporphyrin IX were purchased from Frontier Scientific (Logan, UT). Nonionic detergents decyl maltoside $\left(\mathrm{C}_{10} \mathrm{M}\right)$ and polyoxyethylene(6)decyl ether $\left(\mathrm{C}_{10} \mathrm{E}_{6}\right)$ were purchased from Affymetrix (Santa Clara, $\mathrm{CA}$ ), while n-octyl- $\beta$-D-glucopyranoside ( $\beta \mathrm{OG}$ ) was purchased from Inalco Pharmaceuticals (San Luis Obispo, CA). Zwitterionic detergent 3-[(3-Cholamidopropyl)dimethylammonio]-1propanesulfonate (CHAPS) was purchased from Affymetrix. Tween-20, HPLC-grade chloroform $\left(\mathrm{CHCl}_{3}\right)$ and methanol, sodium cholate, Triton X-100, salicylate, and anti-FLAG M2 affinity resin were purchased from Sigma-Aldrich (St. Louis, MO). FLAG peptide was purchased from GenScript (Piscataway, NJ). Membrane scaffold protein 1E3D1(+) in pET-28a was purchased from AddGene (Cambridge, MA). BioBeads SM-2 was purchased from Bio-Rad (Hercules, MA). The QuikChange ${ }^{\mathrm{TM}}$ II mutagenesis kit was purchased from Agilent Technologies (Santa Clara, CA). Oligos for site-directed mutagenesis were purchased from Integrated DNA Technologies (Coralville, IA). The Bac-to-Bac® Baculovirus expression kit, Spodoptera frugiperda 21 (Sf21) insect cells, fetal bovine serum, 
Fungizone, penicillin-streptomycin, and Sf-900 III serum free media were purchased from Invitrogen (Carlsbad, CA). The phospholipid 1-palmitoyl-2-oleoyl-sn-glycero-3phosphocholine (POPC) was purchased from Avanti Polar Lipids (Alabaster, AL). Methylene chloride $\left(\mathrm{CH}_{2} \mathrm{Cl}_{2}\right)$ was purchased from VWR (Radnor, PA). Acetic acid and formic acid were purchased from Fisher Scientific (Waltham, MA). Ethyl acetate was purchased from J.T. Baker (Phillipsburg, NJ). Citric acid was purchased from USB Corporation (Cleveland, $\mathrm{OH})$.

\section{Engineering, expression, and solubilization of COX-2 constructs}

Wild type, S530T, and G533V murine (mu) COX-2 constructs were prepared as described in ${ }^{29}$. The S530T/G533V double mutant was generated using G533V muCOX-2 in pFastBac-1 as the template for mutagenesis. His ${ }_{6}$ N580A human (hu) COX-2 in pFastBac-1 was used to engineer FLAG huCOX-2 using the protocol described in ${ }^{30}$. The resulting FLAG huCOX-2 construct then served as a template for the deletion of residues 586-612 $(\Delta 586)$ at the C-terminus ${ }^{31}$. The last six residues of huCOX-2 (Glu-613 through Leu-618) were retained in the construct. All constructs were sequence verified. Expression was carried out in insect cells as described in ${ }^{7}$. For solubilization, the cell pellet from a $2 \mathrm{~L}$ culture of Sf 21 cells was resuspended in $50 \mathrm{mM}$ Tris, $\mathrm{pH} 8.0,300 \mathrm{mM} \mathrm{NaCl}$. The resuspended cells were lysed using a Microfluidizer and solubilized by adding one of four detergents, followed by stirring for $1 \mathrm{hr}$ at $4{ }^{\circ} \mathrm{C}: \mathrm{C}_{10} \mathrm{M}$ to a final concentration of $0.87 \%(\mathrm{w} / \mathrm{v})$; Tween-20 to a final concentration of $1 \%(\mathrm{w} / \mathrm{v}) ; \mathrm{C}_{10} \mathrm{E}_{6}$ to a final concentration of $0.8 \%(\mathrm{w} / \mathrm{v})$; or CHAPS to a final concentration of $1.1 \%(\mathrm{w} / \mathrm{v})$. The solubilization mixture was centrifuged at $140,000 \times$ $\mathrm{g}$ for $75 \mathrm{~min}$ and the supernatant was subsequently filtered using a $0.2 \mu \mathrm{m}$ syringe filter prior to the initiation of chromatographic purification procedures.

\section{Purification of COX-2 in different detergent systems and nanodiscs}

Wild type, S530T, G533V, and S530T/G533V muCOX-2 constructs were purified as described in ${ }^{29}$. A two-step purification protocol, consisting of affinity and size-exclusion chromatographic steps, was utilized to generate huCOX-2 in the four detergent systems that have been commonly utilized to biochemically characterize COX-2: $\beta O \mathrm{OG}^{7}, \mathrm{C}_{10} \mathrm{E}_{6}{ }^{16}$, Tween-20 ${ }^{32}$, and CHAPS ${ }^{33}$. The His 6 huCOX-2 construct was used to produce purified protein utilizing the protocol outlined in ${ }^{7}$, with minor modifications. Specifically, wash buffers B and C used for immobilized metal affinity chromatography had imidazole present at concentrations of $77 \mathrm{mM}$ and $250 \mathrm{mM}$, respectively. The detergent concentration in each buffer after size-exclusion chromatography was $0.53 \%$ (w/v) $\beta O G, 0.1 \%$ (w/v) Tween-20, $0.1 \%(\mathrm{w} / \mathrm{v}) \mathrm{C}_{10} \mathrm{E}_{6}$, or $0.5 \%(\mathrm{w} / \mathrm{v})$ CHAPS. The FLAG huCOX-2 construct was used to produce purified protein in $\beta O G$ and nanodiscs utilizing the protocols outlined in ${ }^{30}$. After the affinity chromatography step, half of the sample was used to proceed through the sizeexclusion chromatography step described above ${ }^{7}$. Incorporation of FLAG huCOX-2 into nanodiscs comprised of POPC phospholipids was carried out as described in ${ }^{30}$. The FLAG $\Delta 586$ huCOX-2 construct was utilized to generate protein in $\beta O G$ for crystallography by combining the FLAG affinity chromatographic step described in ${ }^{30}$ and the size-exclusion chromatographic step described in ${ }^{7}$. The peak fractions from the size-exclusion column were pooled and concentrated to $4.3 \mathrm{mg} / \mathrm{mL}$ for crystallization trials. 


\section{Crystallography}

Concentrated S530T muCOX-2 and wild type huCOX-2, both in 25mM Tris, pH 8.0, $150 \mathrm{mM} \mathrm{NaCl}, 0.53 \%(\mathrm{w} / \mathrm{v}) \beta \mathrm{OG}$, were reconstituted with either 2 -fold molar excess of $\mathrm{Fe}^{3+}$-protoporphyrin IX or $\mathrm{Co}^{3+}$-protoporphyrin IX as described in ${ }^{7}$. Crystallization trials were set up at $23{ }^{\circ} \mathrm{C}$ using the sitting drop vapor diffusion method. $3 \mu \mathrm{L}$ of protein solution was combined with $3 \mu \mathrm{L}$ of a drop solution consisting of $23-34 \%$ polyacrylic acid 5100 , 100mM HEPES, pH 7.5, 20mM $\mathrm{MgCl}_{2}$, and $0.6 \%(\mathrm{w} / \mathrm{v}) \beta \mathrm{OG}$ and equilibrated over $0.5 \mathrm{~mL}$ reservoir solution of 23-34\% polyacrylic acid 5100, 100mM HEPES, pH 7.5, and 20mM $\mathrm{MgCl}_{2}$. Crystals were subsequently harvested from the drop and soaked for $30 \mathrm{~min}$ in $33 \%$ polyacrylic acid 5100, 100mM HEPES, pH 7.5, $20 \mathrm{mM} \mathrm{MgCl}_{2}$, and $0.6 \%(\mathrm{w} / \mathrm{v}) \beta O G$ that was supplemented with either $500 \mu \mathrm{M}$ ASA or $500 \mu \mathrm{M}$ salicylate (SAL) and $10 \%$ ethylene glycol for cryopreservation. Cryoprotected crystals were then looped and frozen directly in a gaseous nitrogen stream cooled to $100 \mathrm{~K}$. Diffraction data for the huCOX-2 structures were collected at the Advanced Photon Source (Argonne, IL). Specifically, data for aspirinacetylated FLAG $\triangle 586$ huCOX-2 (huCOX2:ASA) were collected on beamline 23ID-B utilizing a MAR mosaic 300 CCD detector, while data for FLAG $\triangle 586$ huCOX-2 complexed with SAL were collected on beamline 17ID-B utilizing a Dectris Pilatus 6M pixel array detector. The diffraction data for the huCOX2 structures were reduced, integrated, and scaled using iMOSFLM ${ }^{34}$ and AIMLESS ${ }^{35}$ from the CCP4 suite of programs ${ }^{36}$. Diffraction data for S530T muCOX-2 were collected on beamline A1 at the Cornell High Energy Synchrotron Source using an Area Detector Systems CCD Quantum-210 detector. The S530T muCOX-2 dataset was integrated and scaled using MOSFLM and SCALA in the CCP4 suite of programs. Data collection statistics are detailed in Table 1.

The structure of S530T muCOX-2 was solved utilizing molecular replacement methods coupled with the protocol detailed in ${ }^{7}$. Iterative rounds of model building and refinement were carried out in COOT ${ }^{37}$ and PHENIX ${ }^{38}$ to place waters and ligands. Translationlibration-screw (TLS) refinement ${ }^{39}$, utilizing automated TLS group determination in PHENIX, was carried out in the final rounds of refinement.

The structures of huCOX-2:ASA and huCOX-2:SAL were solved by molecular replacement methods using a truncated search model of muCOX-2 derived from PDB entry $3 \mathrm{HS} 5{ }^{7}$ and PHASER ${ }^{40}$. The truncated search model had all of its ligands, cofactors, and waters deleted, as well as membrane binding domain residues 33-144, and catalytic domain residues 275282, 342-393, and 504-540. The initial maps generated by PHASER were subject to density modification by PARROT, followed by submission to the BUCCANEER pipeline, both in the CCP4 suite of programs ${ }^{41}$, for automated model building. The huCOX-2 primary sequence was input as part of the automated building process to facilitate changing the nonidentical residues between the muCOX-2 and huCOX-2 models. BUCCANEER successfully built $95 \%$ of the huCOX-2 model for each structure. Iterative cycles of model building in COOT and refinement using PHENIX was employed to complete the protein models and place all remaining ligands, cofactors, and waters. TLS refinement, utilizing automated TLS group determination in PHENIX, was carried out in the final rounds of refinement. 
The refined models of huCOX-2:ASA, huCOX-2:SAL, and S530T muCOX-2 contain one sequence homodimer in the asymmetric unit. Each monomer is comprised of residues 33583, $\mathrm{Co}^{3+}$-protoporphyrin IX, and carbohydrate moieties linked to Asn-68, Asn-144, and Asn-410. The huCOX-2:ASA structure has an acetylated Ser-530 side chain modeled within the cyclooxygenase channel of each monomer, while the huCOX2:SAL structure has a molecule of SAL modeled within the cyclooxygenase channel of each monomer. We modeled a pluronic F-68 molecule, derived from the insect cell expression media, in the cyclooxygenase channel of each monomer of the S530T muCOX-2 structure. Refinement statistics are detailed in Table 1. Simulated annealing omit maps were generated using PHENIX and model validation was carried out using MOLPROBITY ${ }^{42}$. Figures were produced using PYMOL (Version 1.7.0.0; Schrodinger, LLC). Coordinates and structure factors for huCOX-2:ASA, huCOX-2:SAL, and S530T muCOX-2 have been deposited in the protein data bank (PDB id 5F19, 5F1A, and 5FDQ respectively).

\section{Activity assays}

Peroxidase and cyclooxygenase assays were performed as described in ${ }^{7}$. For inhibition studies with ASA, huCOX-2 at a concentration of $5 \mu \mathrm{M}$ was incubated with $500 \mu \mathrm{M}$ ASA at room temperature, followed by the measurement of cyclooxygenase activity over the course of 150 minutes. For inhibition studies with other NSAIDs, $5 \mu \mathrm{M}$ huCOX-2 was incubated with $100 \mu \mathrm{M}$ ibuprofen or $50 \mu \mathrm{M}$ flurbiprofen or $50 \mu \mathrm{M}$ celecoxib on ice. In the case of ibuprofen, $100 \mu \mathrm{M}$ inhibitor was also added to the reaction cuvette prior to initiating the cyclooxygenase reaction. Cyclooxygenase activity was subsequently measured at designated time points over the course of 10 minutes. The concentration of each huCOX-2 preparation was determined by an absorbance reading taken at $280 \mathrm{~nm}$. These readings were verified by running the protein out on an SDS-PAGE gel alongside bovine serum albumin loaded at known concentrations, followed by densitometric analysis of the protein bands using Image $\mathrm{J}^{43}$.

\section{Product analysis}

Radio-thin layer chromatography (TLC) was used to analyze the products generated from the incubation of $\left[1-{ }^{14} \mathrm{C}\right] \mathrm{AA}$ with wild type and aspirin-acetylated huCOX-2. To prepare the samples for TLC, $5 \mu \mathrm{M}$ huCOX-2 was incubated with either 500 $\mu \mathrm{M}$ ASA or vehicle (ethanol) at room temperature for $150 \mathrm{~min}$. The proteins were subsequently mixed with $\left[1-{ }^{14} \mathrm{C}\right] \mathrm{AA}$ and incubated for 75 seconds at $37^{\circ} \mathrm{C}$. The final concentrations of huCOX-2 and $\left[1-{ }^{14} \mathrm{C}\right] \mathrm{AA}$ were $1 \mu \mathrm{M}$ and $50 \mu \mathrm{M}$, respectively. The reactions were quenched by the addition of a 2-fold volume excess of $\mathrm{CHCl}_{3}: \mathrm{MeOH}: 200 \mathrm{mM}$ citrate (30:4:1), mixing thoroughly, and placing the samples on ice. The samples were subsequently microfuged and the organic phase was spotted onto a $10 \mathrm{~cm} \times 10 \mathrm{~cm}$ high-performance TLC plate (Merck), with a $10 \mathrm{~cm}$ $\times 2.5 \mathrm{~cm}$ concentrating zone. The plate was developed in a chamber containing $\mathrm{CH}_{2} \mathrm{Cl}_{2}$ :ethyl acetate:formate:acetate (84:12:1:1). Once dried, the plate was exposed to a phosphor screen for $24 \mathrm{hr}$. The phosphor screen was then imaged using a Typhoon imager and the density of the radioactive bands was quantified with ImageJ. The relative product compositions were calculated based on the relative band intensities co-chromatographing with non-radioactive standards. 


\section{Quantification of acetyl incorporation}

huCOX-2 at a concentration of $5 \mu \mathrm{M}$ was incubated with $500 \mu \mathrm{M}$ [acetyl- ${ }^{14} \mathrm{C}$ ] ASA in a volume of $10 \mu \mathrm{L}$ at room temperature for $150 \mathrm{~min}$. The reaction was quenched by the addition of $5 \mu \mathrm{L}$ Laemmli sample buffer containing $\beta \mathrm{ME}$ and heating at $70^{\circ} \mathrm{C}$ for $10 \mathrm{~min}$. The sample was loaded on a 4-20\% polyacrylamide gel and run for $35 \mathrm{~min}$ at $120 \mathrm{~V}$. Following destaining, the gel was soaked in a 5\% glycerol solution and dried in a gel-dryer apparatus. The dried gel was exposed to the phosphor screen for $24 \mathrm{hr}$. The screen was then imaged using a Typhoon imager and the densities of the radioactive bands were determined using ImageQuant (GE Healthcare).

\section{RESULTS}

\section{Crystal structures of acetylated and salicylate bound human COX-2}

For structural characterization of huCOX-2, a crystallization construct was generated that utilized a FLAG tag just beyond the signal sequence at the $\mathrm{N}$-terminus of the enzyme to facilitate purification ${ }^{30}$. In addition, we deleted residues 586-612 at the C-terminus of the enzyme ( $\Delta 586)$, which corresponds to the 27 amino acid instability motif involved in the degradation of COX-2 ${ }^{31,44}$. These residues were not observed in the previously published crystal structures of huCOX-2 ${ }^{45}$. Moreover, these residues have historically been removed via trypsin digestion during purification prior to the generation of crystals of muCOX-2 $7,29,46$. The resulting FLAG $\Delta 586$ huCOX-2 construct retained wild-type levels of peroxidase and cyclooxygenase activity (data not shown). Prior to crystallization, FLAG $\triangle 586$ huCOX-2 was reconstituted with a 2-fold molar excess of either $\mathrm{Fe}^{3+}$-protoporphyrin IX or $\mathrm{Co}^{3+}$-protoporphyrin IX. Both reconstituted enzymes were readily crystallized. However, $\mathrm{Co}^{3+}$-protoporphyrin IX reconstituted FLAG $\Delta 586$ huCOX-2 generated more single and larger crystals that were easier to manipulate and that diffracted to higher resolutions than the $\mathrm{Fe}^{3+}$-protoporphyrin IX reconstituted enzyme.

The crystal structures of acetylated FLAG $\Delta 586$ huCOX-2 (huCOX-2:ASA) and salicylate bound FLAG $\triangle 586$ huCOX-2 (huCOX-2:SAL) were solved to resolutions of $2.04 \AA$ and $2.38 \AA$, respectively, using synchrotron radiation and molecular replacement methods. Both structures crystallized in space group I222 and contain the canonical sequence homodimer in the asymmetric unit, analogous to what was observed previously for huCOX-2 ${ }^{45}$. There are no significant differences observed when the two monomers of the homodimer are superimposed, with calculated root mean square deviations (r.m.s.d) between monomers of $0.325 \AA$ and $0.277 \AA$ for huCOX-2:ASA and huCOX-2:SAL, respectively. Furthermore, there are no significant differences within the monomers when they are compared to monomers in the crystal structures of muCOX-2 complexed with fatty acid and endocannabinoid substrates $7,29,46$. As expected, the $\mathrm{N}$-terminal epidermal growth factor-like domain, the membrane-binding domain (MBD), and the C-terminal catalytic domain are well resolved in each monomer and conserved between muCOX-2 and huCOX-2.

We observe acetylation on the side chain of Ser-530 in both monomers of the huCOX-2:ASA crystal structure (Figure 1A; Suppl. Figure 1A). Moreover, the spatial arrangement of the acetylated Ser-530 side chain at the apex of the cyclooxygenase channel 
is identical in each monomer. The acetyl moiety makes a total of four contacts with residues lining the channel (Suppl. Table 1). The carbonyl oxygen of the acetyl moiety forms a hydrogen bond with the phenolic oxygen of Tyr-385, while the $\mathrm{C} 2$ atom makes three hydrophobic contacts with carbon atoms on the side chains of Val-344, Tyr-348, and Val-349 (Figure 1B). Collectively, these interactions stabilize the acetylated side chain within the active site channel and effectively block access to the hydrophobic groove above Ser-530.

SAL binds in the cyclooxygenase channel just below the side chain of Tyr-385 and adjacent to the side chain of Ser-530 (Figure 1C; Suppl. Figure 1B). The contacts made by SAL within the channel are hydrophobic in nature (Suppl. Table 2). In the observed configuration, the hydroxyl moiety of SAL points towards the hydroxyl group of the side chain of Ser-530, which lies 3A away. Carbons C3, C4, and C5 of SAL make a total of 11 hydrophobic contacts with the side chains of Leu-384, Tyr-385, and Trp-387 located at the apex of the channel. The carboxylate moiety of SAL does not interact with any of the residues lining the cyclooxygenase channel, which explains why SAL has a weak binding affinity for $\mathrm{COX}-2{ }^{47}$.

Loll and colleagues determined the structure of ovine (ov) COX-1 inactivated by a brominated aspirin analogue to $3.4 \AA$ resolution ${ }^{19}$. The structure details the molecular interactions for both the acetylated Ser-530 side chain and the SAL leaving group within the cyclooxygenase channel of ovCOX-1. When compared to the huCOX2:ASA crystal structure, the hydrogen-bonding network between the acetylated Ser-530 side chain and the phenolic oxygen atoms of Tyr-348 and Tyr-385 is completely conserved, although the presence of the bromine atom in the ovCOX-1 crystal structure results in a different rotamer conformation for Ser-530 (Figure 2A). There is a $\sim 5 \AA$ shift in location of SAL within the cyclooxygenase channel in the huCOX-2:SAL crystal structure compared to its location in the ovCOX-1 cyclooxygenase channel (Figure 2B). The carboxylate of the SAL leaving group in the ovCOX-1 structure interacts with the side chains of Arg-120 and Tyr-355 at the opening of the channel, similar to that observed for carboxylate-containing NSAIDs 48,49 . The conformation of SAL observed within the cyclooxygenase channel in the huCOX-2:SAL crystal structure suggests that this is a pose reflective of the enzymeinhibitor complex prior to acetylation. However, it could also represent a pose reflective of the leaving group after acetylation.

\section{The role of side chain flexibility in the binding of AA to acetylated COX-2}

Previous mutational studies have shown that in aspirin-acetylated COX-2, the $\omega$-end of AA requires access to the hydrophobic groove to generate $15 \mathrm{R}-\mathrm{HETE}^{27}$. The crystal structure of huCOX2:ASA presented here indicates that access to the hydrophobic groove is completely blocked upon acetylation of Ser-530. Thus, the acetylated Ser-530 side chain must exist in a different conformation during the generation of 15R-HETE compared to the conformation observed in the crystal structure. To this end, crystal structures of ovCOX-1 and muCOX-2 elucidated in complex with substrates indicate that the side chain of Ser-530 can take on dual conformations depending on the type of substrate bound and the substrate's mode of binding within the cyclooxygenase channel ${ }^{7,29,46,50}$. Indeed, we initially proposed that the 
observed flexibility for the Ser-530 side chain facilitates access of the $\omega$-end of the substrate to the hydrophobic groove and potentially plays a role in the generation of 15R-HETE upon aspirin acetylation ${ }^{51}$. In addition to its role in the aspirin-acetylation reaction, Ser-530 is a critical player in the maintenance of the proper stereochemistry for oxygen addition at carbon-15 during the production of $\mathrm{PGG}_{2}$. Substitution of Ser-530 with threonine in ovCOX-1 produces an active enzyme that behaves like aspirin-acetylated COX-2, producing 15R-HETE exclusively ${ }^{52}$. Similarly, replacement of Ser-530 with threonine or methionine in COX-2 results in an almost complete shift in product stereochemistry at carbon-15 towards the R stereoisomer ${ }^{24}$.

We evaluated S530T muCOX-2, along with the S530T/G533V muCOX-2 double mutant, to determine if the S530T mutation also restricted the binding of the $\omega$-end of AA within the hydrophobic groove similar to that observed upon aspirin-acetylation. Gly-533 is located at the end of the hydrophobic groove, where it makes numerous contacts with carbons 17-19 of AA ${ }^{7}$. G533V COX-2 is devoid of cyclooxygenase activity, as the $\omega$-end of AA cannot fully access the hydrophobic groove, leading to the misalignment of carbon-13 below Tyr-385 ${ }^{29}$. Therefore, combining the G533V mutation with the S530T mutation provides insight on the binding pose of AA within the cyclooxygenase channel of COX-2 that leads to the formation of $15 \mathrm{R}-\mathrm{PGG}_{2}$. We engineered S530T and the S530T/G533V double mutant and carried out kinetic analyses utilizing AA as the substrate. AA is oxygenated by S530T muCOX-2 at $24 \%$ the rate of wild type enzyme, with a calculated $\mathrm{K}_{\mathrm{M}}$ value of $6.5 \pm 0.4 \mu \mathrm{M}$. In contrast, S530T/G533V muCOX-2 did not retain any cyclooxygenase activity, indicating that the $\omega$-end of AA must also access the hydrophobic groove to generate $15 \mathrm{R}-\mathrm{PGG}_{2}$.

To complement our functional studies, we determined the crystal structure of S530T muCOX-2 to $1.9 \AA$ resolution. The structure contains a sequence homodimer within the asymmetric unit and there are no significant differences in the tertiary structure observed when the monomers are compared to one another. Superposition of the S530T muCOX-2 crystal structure onto the wild type structure complexed with AA (PDB id $3 \mathrm{HS} 5 ;{ }^{7}$ ) provides insight into how the S530T mutation would influence the binding of AA within the hydrophobic groove. Interestingly, the observed Thr-530 side chain rotamer perfectly overlays with the alternate conformation observed for the Ser-530 side chain in the wild type structure complexed with AA (Figure 3). Hence, there is ample room for the $\omega$-end of the substrate to access the hydrophobic groove in the S530T mutant, which is in agreement with both our kinetic analyses and the previously observed production of $15 \mathrm{R}$ PG products by this mutant ${ }^{24}$.

\section{Functional characterization of human COX-2 in various solubilization systems}

It is well established that the treatment of COX-2 with aspirin results in the acetylation of the Ser-530 side chain. As COX enzymes are sequence homodimers, there are two potential acetylation sites, one located within the cyclooxygenase channel of each monomer. As observed in the huCOX-2:ASA crystal structure, the expectation is that aspirin is nondiscriminant with respect to the acetylation of each Ser-530 side chain. Contrary to the crystallographic results presented here, recent studies provide evidence for the acetylation of Ser-530 in only a single monomer of COX-2 22,53 . One of the major differences between 
studies across laboratories has been the detergent utilized to solubilize and purify COX-2 for subsequent analyses of aspirin acetylation.

We utilized huCOX-2 that was solubilized and purified in four different detergent systems ( $\beta O G, \mathrm{C}_{10} \mathrm{E}_{6}$, Tween-20, and CHAPS), in addition to reconstituting huCOX-2 into nanodiscs, to evaluate the effects that the choice of detergent/lipid system used to stabilize COX-2 had on catalysis and inhibition. The four detergents, along with nanodisc reconstitution represent systems that have been previously utilized to characterize the biophysical and functional properties of COX-2 16, 30, 32, 33. For each system,

cyclooxygenase activity was measured using an oxygen electrode, with AA as the substrate, while peroxidase activity was measured spectrophotometrically using $\mathrm{N}, \mathrm{N}, \mathrm{N}^{\prime}, \mathrm{N}^{\prime}-$ tetramethylphenylenediamine and $\mathrm{H}_{2} \mathrm{O}_{2}$. Not surprisingly, each system successfully maintained both the cyclooxygenase and peroxidase activity of huCOX-2 (Table 2). In addition, the values obtained for $\mathrm{k}_{\text {cat }}$ and $\mathrm{K}_{\mathrm{M}}$ were similar between the five systems and in line with values reported previously for huCOX-2 ${ }^{53}$.

We next evaluated the inhibition of huCOX-2 in each of the five systems. The nonselective NSAIDs ibuprofen and flurbiprofen, along with the COX-2 selective inhibitor celecoxib were utilized for these studies. These inhibitors are representatives of time-independent and time-dependent modes of COX inhibition. Overall, huCOX-2 in the different systems exhibited similar inhibition profiles for all of the compounds tested (Figure 4). The magnitude of inhibition by ibuprofen was virtually identical for all five systems tested. Similarly, time-dependent inhibition by flurbiprofen and celecoxib reached similar levels in each system after 30 minutes, with huCOX-2 in CHAPS and Tween-20 exhibiting a slower rate of inhibition when tested with flurbiprofen and celecoxib, respectively. Collectively, each of the five systems utilized to stabilize huCOX-2 did not affect the ability of the enzyme to oxygenate AA or to be inhibited by ibuprofen, flurbiprofen, or celecoxib.

Lastly, we evaluated the ability of huCOX-2 to be acetylated by aspirin in each of the five systems. huCOX-2 in each system was incubated with $500 \mu \mathrm{M}$ aspirin at room temperature and aliquots were removed in 30-minute intervals over the course of 150 minutes, followed by measurement of the remaining oxygenase activity. To supplement these measurements, we quantified the amount of acetylated huCOX-2 generated using [acetyl- ${ }^{14} \mathrm{C}$ ]ASA coupled with SDS-PAGE analysis and characterized product profiles using $\left[1-{ }^{14} \mathrm{C}\right] \mathrm{AA}$ coupled with thin layer chromatography. The results are depicted in Figure 5. Unlike the kinetic and inhibition profiles described above, the levels of aspirin acetylation varied when the different systems were compared. huCOX-2 in $\beta O G$ and CHAPS, along with nanodiscreconstituted enzyme resulted in a decrease in oxygenase activity upon acetylation to $\sim 10 \%$ of the starting activity after 150 minutes. Conversely, acetylated huCOX-2 in $\mathrm{C}_{10} \mathrm{E}_{6}$ retained $\sim 60 \%$ oxygenase activity over the $150 \mathrm{~min}$ time course. Interestingly, huCOX-2 in Tween-20 was not acetylated by aspirin treatment. Quantification of radioactive acetyl incorporation and product profile analyses of huCOX-2 in the five systems are also consistent with the amount of oxygenase activity remaining after 150 minutes (Figure 5BD). Our results indicate that there is differential acetylation of huCOX-2 that is dependent upon the detergent system utilized to stabilize the purified enzyme. Moreover, these results 
are consistent with previously published studies that indicate differential levels of aspirin acetylation of COX-2 reconstituted in $\mathrm{C}_{10} \mathrm{E}_{6}, \beta \mathrm{OG}$, and nanodiscs ${ }^{22,} 30$.

\section{DISCUSSION}

Aspirin acetylation of COX-1 and COX-2 leads to differential effects on each isoform's ability to oxygenate AA. While the acetylation of COX-1 leads to complete loss of cyclooxygenase activity, acetylation of COX-2 results in the production of 15R-HETE from AA. The structural and mechanistic basis of COX-1 inhibition by aspirin is well established, with the acetylation of Ser-530 at the apex of the cyclooxygenase channel resulting in the blockage of the productive binding of AA required for the generation of product ${ }^{19}$. In contrast, for COX-2 little is known at the molecular level as to how the acetylated Ser-530 side chain is accommodated within the cyclooxygenase channel and what steric affect this bulky side chain has on the binding pose of AA. The crystal structures and functional data presented here provide a first look at the atomic level details associated with the acetylation reaction in COX-2 and allow for a hypothesis to be put forth related to the binding of AA in the acetylated active site that leads to the generation of 15R-HETE.

There is clear electron density for an acetylated Ser-530 side chain in each monomer of the huCOX-2:ASA crystal structure. In both monomers, the acetylated Ser-530 side chain adopts a conformation that allows for a hydrogen-bonding network to be formed between the carbonyl oxygen of the acetyl group, the phenolic oxygen of Tyr-385, and the phenolic oxygen of Tyr-348. The importance of this network was exemplified using mutagenesis studies, in which Y348F or Y385F COX-2 constructs resulted in diminished acetyl incorporation upon ASA treatment ${ }^{54}$. It was proposed that these residues play a critical a role in stabilizing the incipient negative charge of the tetrahedral intermediate produced during the acetylation reaction. The distances observed in the huCOX2:ASA crystal structure support this potential reaction scheme.

Insertion of the $\omega$-end of the substrate into the hydrophobic groove is responsible for proper bisallylic carbon positioning below Tyr-385, which ultimately drives efficient substrate oxygenation by COX-2 ${ }^{29}$. Examination of the acetylated COX-2 cyclooxygenase channel and comparison to wild type crystal structures of COX-2 in complex with substrates clearly indicates that the rotamer adopted by acetylated Ser-530 prohibits entry of the $\omega$-end of the substrate into the hydrophobic groove (Figure 3B). One of the hallmark differences between $\mathrm{COX}$ isoforms is the larger volume associated with the cyclooxygenase channel of COX-2 due to substitutions of residues Ile-434, His-513, and Ile-523 in COX-1 to Val-434,

Arg-513, and Val-523 in COX-2. Given the increased volume, it was proposed that AA could adopt a "hairpin" conformation in the acetylated cyclooxygenase channel of COX-2 instead of the canonical L-shaped conformation observed for productive binding leading to $\mathrm{PGG}_{2}$ formation. In the hairpin conformation, carbon- 13 of AA would still be aligned below Tyr-385, but the $\omega$-end would fold back on the carboxylate end of the substrate ${ }^{55}$. The binding of AA in the hairpin conformation would also explain how Ser-530 controls the stereospecificity of oxygen addition at carbon-15 as observed in the generation of 15R-PG products by S530T and S530M COX-2 constructs ${ }^{24}$. The finding that the S530T/G533V double mutant does not retain cyclooxygenase activity indicates that the $\omega$-end of AA must 
bind within the hydrophobic groove to generate 15R-PGs. As previous studies also indicate that acetylated G533V COX-2 is inactive ${ }^{27}$, a prerequisite for the formation of 15R-HETE and 15R-PG products is that AA binds in the canonical L-shaped conformation within the cyclooxygenase channel.

If the $\omega$-end of AA is required to bind within the hydrophobic groove, then the observed conformation of acetylated Ser-530 in the huCOX2:ASA crystal structure must not represent the conformation required to generate 15R-HETE. Tosco recently utilized molecular dynamics to generate models of AA bound to acetylated COX-2 ${ }^{25}$. The simulations suggest that the acetylated Ser-530 side chain adopts an alternate conformation that would allow AA to bind in an L-shaped conformation within the hydrophobic groove. Significant efforts were made to determine the crystal structure of AA bound within the acetylated cyclooxygenase channel of COX-2 to no avail. Thus, we subsequently utilized the experimentally derived huCOX2:ASA crystal structure in conjunction with the crystal structure of AA bound to wild type COX-2 to examine how an alternate rotamer of the acetylated Ser-530 side chain facilitates substrate binding. As observed in the molecular dynamics simulations, rotating the acetylated side chain such that the acetyl group is directed toward the constriction site of the cyclooxygenase channel facilitates access of the $\omega$-end of AA to the hydrophobic groove (Figure 6). The resulting pose of AA satisfies the requirements for hydrogen abstraction, with carbon-13 positioned beneath Tyr-385. However, the alternate conformation of the acetylated Ser-530 side chain would force AA into a more extended state, disfavoring endoperoxide cyclization and straining the pentadienyl radical, leading to the trapping of the allyl radical on carbon- 15 .

The seeming restriction to the alternate Ser-530 side chain rotamer is the presence of the side chain of Leu-531, which lies just below Ser-530 in the cyclooxygenase channel. Presumably, the steric clash between the Leu-531 and acetylated Ser-530 side chains would prohibit such a rotamer from existing. However, numerous crystal structures of COX-2 in complex with substrates and inhibitor indicate that the Leu-531 side also exhibits conformational flexibility such that it can be flipped away from the constriction site at the channel entrance $7,29,46,56$. The alternate positioning of the Leu-531 side chain would facilitate the movement of the acetylated Ser-530 side chain required for the optimal binding of AA within the acetylated cyclooxygenase channel (Figure 6). The observed flexibility for the Leu-531 side chain is unique to COX-2 7,46 and has not yet been observed in crystal structures of COX-1. However, previous studies have shown that mutations to Leu-531 are more tolerated in COX-2 than COX-1, suggesting that the rotamer conformation for Leu-531 observed in COX-1 crystal structures is required to stabilize the enzyme for optimal substrate oxygenation ${ }^{7,52}$. Therefore, it is intriguing to speculate that the differential effect observed upon aspirin acetylation of COX-1 and COX-2 may in part be due to the inability of COX-1 to adopt the proposed alternate acetyl-Ser-530 conformation.

While the solubilization system employed to stabilize purified COX-2 in solution has little effect on the inhibition of the enzyme by ibuprofen, flurbiprofen, and celecoxib, we observe significant differences in the rate and completeness of the acetylation reaction. The underlying mechanism responsible for these differences is not clear, although several possibilities exist including: 1 ) aspirin is more prone to sequestration within the micelles of 
certain detergents; 2) aspirin is hydrolyzed more rapidly in different detergent buffers, thus decreasing the effective concentration in solution; or 3) the detergents interact with COX-2 differently resulting in differential access of aspirin to the cyclooxygenase channel. No obvious hydrolysis or sequestration was observed in control experiments carried out to measure the breakdown of aspirin in different detergent buffers and evaluate the sequestration of aspirin within detergent micelles (data not shown).

The other NSAIDs tested bind and inhibit COX-2 equivalently across solubilization systems. These NSAIDs presumably enter the cyclooxygenase channel via a similar path and are not affected by the detergent system utilized ${ }^{30}$. If we consider that aspirin traverses through the MBD of the enzyme, differences in how detergents interact with the amphipathic a-helices that make up the MBD may specifically influence the ability of aspirin to enter the cyclooxygenase channel and subsequently acetylate Ser-530. Indeed, detergent molecules have been observed within the MBD in COX crystal structures ${ }^{6,57}$. It may be that some detergents exert an indirect effect on the cyclooxygenase channel opening that alters some structural or electrostatic interaction required for aspirin to access the cyclooxygenase channel. The altered inhibition time course of celecoxib may implicate the region around the COX-2 specific side pocket housing Arg-513 ${ }^{58}$, although there is no precedent to suggest this residue is involved in aspirin inhibition.

In summary, the crystal structures of huCOX2:ASA and huCOX2:SAL provide the first structural snapshots of the interactions made by the acetylated Ser-530 side chain and salicylic acid in the cyclooxygenase channel of COX-2. These experimentally derived structures, along with the crystal structure of S530T muCOX-2 and observations published previously, have led to a working hypothesis that describes the optimal binding mode for AA within the cyclooxygenase channel for the generation of 15R-HETE and 15R-PGs. Our model, which is in agreement with previous molecular dynamics simulations, suggests that the combined flexibility of the Leu-531 and acetylated Ser-530 side chains work in tandem to allow AA to bind in an L-shaped conformation within the cyclooxygenase channel, with the $\omega$-end of the substrate located within the hydrophobic groove. We also observed significant differences in the rate of aspirin acetylation of huCOX-2 that was dependent upon the nature of the detergent or lipid system utilized to stabilize purified enzyme. While the mechanism underlying the differential acetylation of Ser-530 is not clear, detergent binding within the MBD of the enzyme may play a role.

\section{Supplementary Material}

Refer to Web version on PubMed Central for supplementary material.

\section{Acknowledgments}

$\mathrm{X}$-ray diffraction experiments related to the determination of the huCOX2:ASA structure were conducted on the GM/CA CAT beamline 23-ID at the Advanced Photon Source (APS). GM/CA CAT has been funded in whole or in part with Federal funds from the National Cancer Institute (Y1-CO-1020) and the National Institute of General Medical Sciences (Y1-GM-1104). X-ray diffraction experiments related to the determination of the huCOX2:SAL structure were conducted on the IMCA CAT beamline 17-ID at the APS. The IMCA CAT beamline is supported by companies of the Industrial Macromolecular Crystallography Association, through a contract with the HauptmanWoodward Medical Research Institute. Use of the APS was supported by the U.S. Department of Energy, Basic Energy Sciences, Office of Science, under contract No. DE-AC02-06CH11357. X-ray diffraction experiments 
related to the determination of the S530T muCOX-2 structure were conducted at the Cornell High Energy Synchrotron Source (CHESS), supported by National Science Foundation Award DMR-0225180, using the Macromolecular Diffraction at CHESS (MacCHESS) facility, supported by National Institutes of Health Award RR-01646.

Funding Statement: Research supported by NIH Grants R01 GM077176 and R01 GM115386.

\section{The abbreviations used are}

AA arachidonic acid

ASA acetylsalicylic acid

BOG n-octyl- $\beta$-D-glucopyranoside

$\mathbf{C}_{10} \mathbf{E}_{6} \quad$ polyoxyethylene(6)decyl ether

$\mathbf{C}_{\mathbf{1 0}} \mathbf{M} \quad$ decyl maltoside

CHAPS 3-[(3-Cholamidopropyl)dimethylammonio]-1-propanesulfonate

COX-2 cyclooxygenase-2

hu human

MBD membrane-binding domain

mu murine

NSAID nonsterroidal anti-inflammatory drug

nsFA nonsubstrate fatty acid

ov ovine

POPC 1-palmitoyl-2-oleoyl-sn-glycero-3-phosphocholine

SAL salicylate

TLS translation-libration-screw

TLC thin layer chromatography;

\section{REFERENCES}

1. Smith WL, Urade Y, Jakobsson PJ. Enzymes of the cyclooxygenase pathways of prostanoid biosynthesis. Chem Rev. 2011; 111:5821-5865. [PubMed: 21942677]

2. Funk CD. Prostaglandins and leukotrienes: advances in eicosanoid biology. Science. 2001; 294:1871-1875. [PubMed: 11729303]

3. Smyth EM, Grosser T, Wang M, Yu Y, FitzGerald GA. Prostanoids in health and disease. J Lipid Res. 2009; (50 Suppl):S423-S428. [PubMed: 19095631]

4. Blobaum AL, Marnett LJ. Structural and functional basis of cyclooxygenase inhibition. J Med Chem. 2007; 50:1425-1441. [PubMed: 17341061]

5. Marnett LJ. The COXIB experience: a look in the rearview mirror. Annu Rev Pharmacol Toxicol. 2009; 49:265-290. [PubMed: 18851701]

6. Malkowski MG, Ginell SL, Smith WL, Garavito RM. The productive conformation of arachidonic acid bound to prostaglandin synthase. Science. 2000; 289:1933-1937. [PubMed: 10988074]

7. Vecchio AJ, Simmons DM, Malkowski MG. Structural basis of fatty acid substrate binding to cyclooxygenase-2. J Biol Chem. 2010; 285:22152-22163. [PubMed: 20463020] 
8. Tsai AL, Kulmacz RJ. Prostaglandin H synthase: resolved and unresolved mechanistic issues. Arch Biochem Biophys. 2010; 493:103-124. [PubMed: 19728984]

9. Thuresson ED, Lakkides KM, Smith WL. Different catalytically competent arrangements of arachidonic acid within the cyclooxygenase active site of prostaglandin endoperoxide $\mathrm{H}$ synthase-1 lead to the formation of different oxygenated products. J Biol Chem. 2000; 275:8501-8507. [PubMed: 10722687]

10. Xiao G, Chen W, Kulmacz RJ. Comparison of structural stabilities of prostaglandin H synthase-1 and -2. J Biol Chem. 1998; 273:6801-6811. [PubMed: 9506982]

11. Yuan C, Rieke CJ, Rimon G, Wingerd BA, Smith WL. Partnering between monomers of cyclooxygenase-2 homodimers. Proc Natl Acad Sci U S A. 2006; 103:6142-6147. [PubMed: 16606823]

12. Kudalkar SN, Nikas SP, Kingsley PJ, Xu S, Galligan JJ, Rouzer CA, Banerjee S, Ji L, Eno MR, Makriyannis A, Marnett LJ. 13-Methylarachidonic acid is a positive allosteric modulator of endocannabinoid oxygenation by cyclooxygenase. J Biol Chem. 2015; 290:7897-7909. [PubMed: 25648895]

13. Dong L, Vecchio AJ, Sharma NP, Jurban BJ, Malkowski MG, Smith WL. Human cyclooxygenase- 2 is a sequence homodimer that functions as a conformational heterodimer. J Biol Chem. 2011; 286:19035-19046. [PubMed: 21467029]

14. Mitchener MM, Hermanson DJ, Shockley EM, Brown HA, Lindsley CW, Reese J, Rouzer CA, Lopez CF, Marnett LJ. Competition and allostery govern substrate selectivity of cyclooxygenase-2. Proc Natl Acad Sci U S A. 2015; 112:12366-12371. [PubMed: 26392530]

15. Prusakiewicz JJ, Duggan KC, Rouzer CA, Marnett LJ. Differential sensitivity and mechanism of inhibition of COX-2 oxygenation of arachidonic acid and 2-arachidonoylglycerol by ibuprofen and mefenamic acid. Biochemistry. 2009; 48:7353-7355. [PubMed: 19603831]

16. Yuan C, Sidhu RS, Kuklev DV, Kado Y, Wada M, Song I, Smith WL. Cyclooxygenase Allosterism, Fatty Acid-mediated Cross-talk between Monomers of Cyclooxygenase Homodimers. J Biol Chem. 2009; 284:10046-10055. [PubMed: 19218248]

17. Lecomte M, Laneuville O, Ji C, DeWitt DL, Smith WL. Acetylation of human prostaglandin endoperoxide synthase-2 (cyclooxygenase-2) by aspirin. J Biol Chem. 1994; 269:13207-13215. [PubMed: 8175750]

18. Shimokawa T, Smith WL. Prostaglandin endoperoxide synthase. The aspirin acetylation region. J Biol Chem. 1992; 267:12387-12392. [PubMed: 1601897]

19. Loll PJ, Picot D, Garavito RM. The structural basis of aspirin activity inferred from the crystal structure of inactivated prostaglandin H2 synthase. Nat Struct Biol. 1995; 2:637-643. [PubMed: 7552725]

20. Mancini JA, O’Neill GP, Bayly C, Vickers PJ. Mutation of serine-516 in human prostaglandin G/H synthase-2 to methionine or aspirin acetylation of this residue stimulates 15-R-HETE synthesis. FEBS Lett. 1994; 342:33-37. [PubMed: 8143845]

21. Rimon G, Sidhu RS, Lauver DA, Lee JY, Sharma NP, Yuan C, Frieler RA, Trievel RC, Lucchesi BR, Smith WL. Coxibs interfere with the action of aspirin by binding tightly to one monomer of cyclooxygenase-1. Proc Natl Acad Sci U S A. 2010; 107:28-33. [PubMed: 19955429]

22. Sharma NP, Dong L, Yuan C, Noon KR, Smith WL. Asymmetric acetylation of the cyclooxygenase- 2 homodimer by aspirin and its effects on the oxygenation of arachidonic, eicosapentaenoic, and docosahexaenoic acids. Mol Pharmacol. 2010; 77:979-986. [PubMed: 20194532]

23. Schneider C, Pratt DA, Porter NA, Brash AR. Control of oxygenation in lipoxygenase and cyclooxygenase catalysis. Chem Biol. 2007; 14:473-488. [PubMed: 17524979]

24. Schneider C, Boeglin WE, Prusakiewicz JJ, Rowlinson SW, Marnett LJ, Samel N, Brash AR. Control of prostaglandin stereochemistry at the 15 -carbon by cyclooxygenases- 1 and -2 . A critical role for serine 530 and valine 349. J Biol Chem. 2002; 277:478-485. [PubMed: 11677234]

25. Tosco P. A mechanistic hypothesis for the aspirin-induced switch in lipid mediator production by cyclooxygenase-2. J Am Chem Soc. 2013; 135:10404-10410. [PubMed: 23786234]

26. van der Donk WA, Tsai AL, Kulmacz RJ. The cyclooxygenase reaction mechanism. Biochemistry. 2002; 41:15451-15458. [PubMed: 12501173] 
27. Rowlinson SW, Crews BC, Goodwin DC, Schneider C, Gierse JK, Marnett LJ. Spatial requirements for $15-(\mathrm{R})$-hydroxy-5Z,8Z,11Z, 13E-eicosatetraenoic acid synthesis within the cyclooxygenase active site of murine COX-2. Why acetylated COX-1 does not synthesize 15-(R)hete. J Biol Chem. 2000; 275:6586-6591. [PubMed: 10692466]

28. Tsai AL, Palmer G, Wu G, Peng S, Okeley NM, van der Donk WA, Kulmacz RJ. Structural characterization of arachidonyl radicals formed by aspirin-treated prostaglandin H synthase-2. J Biol Chem. 2002; 277:38311-38321. [PubMed: 12167656]

29. Vecchio AJ, Orlando BJ, Nandagiri R, Malkowski MG. Investigating substrate promiscuity in cyclooxygenase-2: the role of Arg-120 and residues lining the hydrophobic groove. J Biol Chem. 2012; 287:24619-24630. [PubMed: 22637474]

30. Orlando BJ, McDougle DR, Lucido MJ, Eng ET, Graham LA, Schneider C, Stokes DL, Das A, Malkowski MG. Cyclooxygenase-2 catalysis and inhibition in lipid bilayer nanodiscs. Arch Biochem Biophys. 2014; 546:33-40. [PubMed: 24503478]

31. Mbonye UR, Yuan C, Harris CE, Sidhu RS, Song I, Arakawa T, Smith WL. Two distinct pathways for cyclooxygenase-2 protein degradation. J Biol Chem. 2008; 283:8611-8623. [PubMed: 18203712]

32. Kulmacz RJ, Wang LH. Comparison of hydroperoxide initiator requirements for the cyclooxygenase activities of prostaglandin H synthase-1 and -2. J Biol Chem. 1995; 270:2401924023. [PubMed: 7592599]

33. Rowlinson SW, Crews BC, Lanzo CA, Marnett LJ. The binding of arachidonic acid in the cyclooxygenase active site of mouse prostaglandin endoperoxide synthase-2 (COX-2). A putative L-shaped binding conformation utilizing the top channel region. J Biol Chem. 1999; 274:2330523310. [PubMed: 10438506]

34. Battye TG, Kontogiannis L, Johnson O, Powell HR, Leslie AG. iMOSFLM: a new graphical interface for diffraction-image processing with MOSFLM. Acta Crystallogr D Biol Crystallogr. 2011; 67:271-281. [PubMed: 21460445]

35. Evans PR, Murshudov GN. How good are my data and what is the resolution? Acta Crystallogr D Biol Crystallogr. 2013; 69:1204-1214. [PubMed: 23793146]

36. Dodson EJ, Winn M, Ralph A. Collaborative Computational Project, number 4: providing programs for protein crystallography. Methods Enzymol. 1997; 277:620-633. [PubMed: 18488327]

37. Emsley P, Cowtan K. Coot: model-building tools for molecular graphics. Acta Crystallogr D Biol Crystallogr. 2004; 60:2126-2132. [PubMed: 15572765]

38. Adams PD, Afonine PV, Bunkoczi G, Chen VB, Davis IW, Echols N, Headd JJ, Hung LW, Kapral GJ, Grosse-Kunstleve RW, McCoy AJ, Moriarty NW, Oeffner R, Read RJ, Richardson DC, Richardson JS, Terwilliger TC, Zwart PH. PHENIX: a comprehensive Python-based system for macromolecular structure solution. Acta Crystallogr D Biol Crystallogr. 2010; 66:213-221. [PubMed: 20124702]

39. Winn MC, Isupov MN, Murshudov G. Use of TLS parameters to model anisotrophic displacements in macromolecular refinement. Acta Crystallogr D Biol Crystallogr. 2000; 57:122133. [PubMed: 11134934]

40. McCoy AJ, Grosse-Kunstleve RW, Adams PD, Winn MD, Storoni LC, Read RJ. Phaser crystallographic software. J Appl Crystallogr. 2007; 40:658-674. [PubMed: 19461840]

41. Winn MD, Ballard CC, Cowtan KD, Dodson EJ, Emsley P, Evans PR, Keegan RM, Krissinel EB, Leslie AG, McCoy A, McNicholas SJ, Murshudov GN, Pannu NS, Potterton EA, Powell HR, Read RJ, Vagin A, Wilson KS. Overview of the CCP4 suite and current developments. Acta Crystallogr D Biol Crystallogr. 2011; 67:235-242. [PubMed: 21460441]

42. Davis IW, Leaver-Fay A, Chen VB, Block JN, Kapral GJ, Wang X, Murray LW, Arendall WB, Snoeyink J 3rd, Richardson JS, Richardson DC. MolProbity: all-atom contacts and structure validation for proteins and nucleic acids. Nucleic Acids Res. 2007; 35:W375-W383. [PubMed: 17452350]

43. Schneider CA, Rasband WS, Eliceiri KW. NIH Image to ImageJ: 25 years of image analysis. Nature methods. 2012; 9:671-675. [PubMed: 22930834] 
44. Mbonye UR, Wada M, Rieke CJ, Tang HY, Dewitt DL, Smith WL. The 19-amino acid cassette of cyclooxygenase-2 mediates entry of the protein into the endoplasmic reticulum-associated degradation system. J Biol Chem. 2006; 281:35770-35778. [PubMed: 17001073]

45. Luong C, Miller A, Barnett J, Chow J, Ramesha C, Browner MF. Flexibility of the NSAID binding site in the structure of human cyclooxygenase-2. Nat Struct Biol. 1996; 3:927-933. [PubMed: 8901870]

46. Vecchio AJ, Malkowski MG. The structural basis of endocannabinoid oxygenation by cyclooxygenase-2. J Biol Chem. 2011; 286:20736-20745. [PubMed: 21489986]

47. Laneuville O, Breuer DK, Dewitt DL, Hla T, Funk CD, Smith WL. Differential inhibition of human prostaglandin endoperoxide $H$ synthases- 1 and -2 by nonsteroidal anti-inflammatory drugs. J Pharmacol Exp Ther. 1994; 271:927-934. [PubMed: 7965814]

48. Kurumbail RG, Stevens AM, Gierse JK, McDonald JJ, Stegeman RA, Pak JY, Gildehaus D, Miyashiro JM, Penning TD, Seibert K, Isakson PC, Stallings WC. Structural basis for selective inhibition of cyclooxygenase-2 by anti-inflammatory agents. Nature. 1996; 384:644-648. [PubMed: 8967954]

49. Orlando BJ, Lucido MJ, Malkowski MG. The structure of ibuprofen bound to cyclooxygenase-2. J Struct Biol. 2015; 189:62-66. [PubMed: 25463020]

50. Thuresson ED, Malkowski MG, Lakkides KM, Rieke CJ, Mulichak AM, Ginell SL, Garavito RM, Smith WL. Mutational and X-ray crystallographic analysis of the interaction of dihomo-gamma linolenic acid with prostaglandin endoperoxide H synthases. J Biol Chem. 2001; 276:1035810365. [PubMed: 11121413]

51. Garavito RM, Malkowski MG, DeWitt DL. The structures of prostaglandin endoperoxide H synthases-1 and -2. Prostaglandins Other Lipid Mediat. 2002; 68-69:129-152.

52. Thuresson ED, Lakkides KM, Rieke CJ, Sun Y, Wingerd BA, Micielli R, Mulichak AM, Malkowski MG, Garavito RM, Smith WL. Prostaglandin endoperoxide H synthase-1: the functions of cyclooxygenase active site residues in the binding, positioning, and oxygenation of arachidonic acid. J Biol Chem. 2001; 276:10347-10357. [PubMed: 11121412]

53. Dong L, Sharma NP, Jurban BJ, Smith WL. Pre-existent asymmetry in the human cyclooxygenase-2 sequence homodimer. J Biol Chem. 2013; 288:28641-28655. [PubMed: 23955344]

54. Hochgesang GP Jr, Rowlinson S, Marnett LJ. Tyrosine-385 is critical for acetylation of cyclooxygenase-2 by aspirin. J Am Chem Soc. 2000; 122:6514-6515.

55. Schneider C, Brash AR. Stereospecificity of hydrogen abstraction in the conversion of arachidonic acid to 15R-HETE by aspirin-treated cyclooxygenase-2. Implications for the alignment of substrate in the active site. J Biol Chem. 2000; 275:4743-4746. [PubMed: 10671506]

56. Xu S, Hermanson DJ, Banerjee S, Ghebreselasie K, Clayton GM, Garavito RM, Marnett LJ. Oxicams Bind in a Novel Mode to the Cyclooxygenase Active Site via a Two-water-mediated Hbonding Network. J Biol Chem. 2014

57. Duggan KC, Walters MJ, Musee J, Harp JM, Kiefer JR, Oates JA, Marnett LJ. Molecular basis for cyclooxygenase inhibition by the non-steroidal anti-inflammatory drug naproxen. J Biol Chem. 2010; 285:34950-34959. [PubMed: 20810665]

58. Blobaum AL, Xu S, Rowlinson SW, Duggan KC, Banerjee S, Kudalkar SN, Birmingham WR, Ghebreselasie K, Marnett LJ. Action at a distance: mutations of peripheral residues transform rapid reversible inhibitors to slow, tight binders of cyclooxygenase-2. J Biol Chem. 2015; 290:12793-12803. [PubMed: 25825493]

59. Afonine PV, Grosse-Kunstleve RW, Chen VB, Headd JJ, Moriarty NW, Richardson JS, Richardson DC, Urzhumtsev A, Zwart PH, Adams PD. phenix.model_vs_data: a high-level tool for the calculation of crystallographic model and data statistics. J Appl Crystallogr. 2010; 43:669676. [PubMed: 20648263]

60. Karplus PA, Diederichs K. Linking crystallographic model and data quality. Science. 2012; 336:1030-1033. [PubMed: 22628654] 

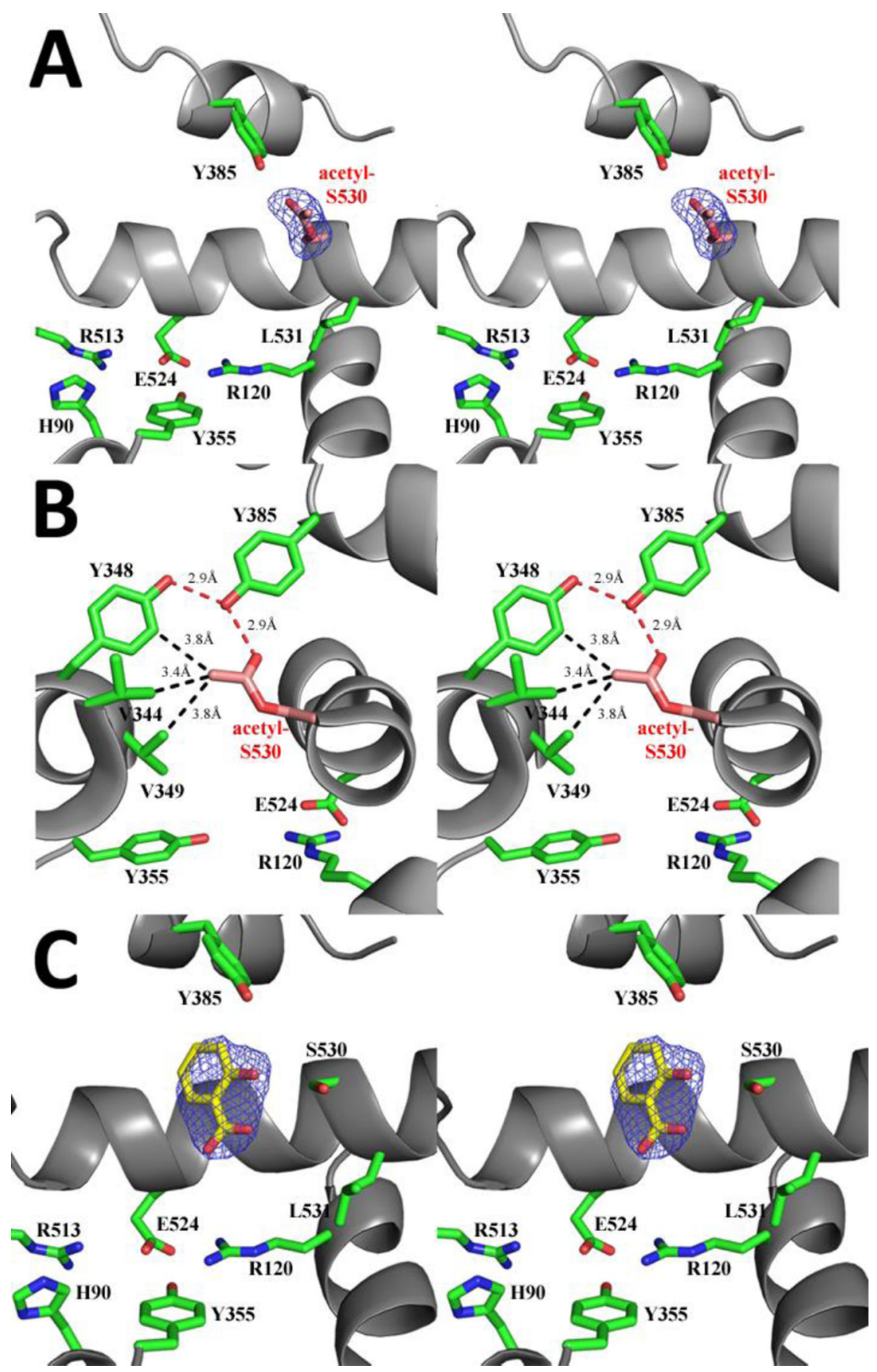

Figure 1. Aspirin-acetylation and Salicylate Binding within the Cyclooxygenase Channel of huCOX-2

(A) Stereo view of the aspirin-acetylated Ser-530 side chain at the apex of the cyclooxygenase channel of monomer $\mathrm{B}$ in the huCOX-2:ASA crystal structure. The $\mathrm{F}_{\mathrm{O}}-\mathrm{F}_{\mathrm{C}}$ simulated annealing omit map electron density (blue), contoured at 3.0 $\sigma$, is shown for the acetylated Ser-530 side chain (pink carbon atoms and red oxygen atoms). (B) Stereo view depicting hydrogen bond (red dashes) and hydrophobic contact (black dashes) distances between the side chains of Val-344, Tyr-348, Val-349, Tyr-385, and acetylated Ser-530. (C) Stereo view of salicylate bound within the cyclooxygenase channel of monomer $\mathrm{A}$ in the 
huCOX-2:SAL crystal structure. The $\mathrm{F}_{\mathrm{O}}-\mathrm{F}_{\mathrm{C}}$ simulated annealing omit map electron density (blue), contoured at $3.0 \sigma$, is shown for salicylate (yellow carbon atoms and red oxygen atoms). Residues lining the cyclooxygenase channel are labeled accordingly, with carbon, nitrogen, and oxygen atoms colored green, blue, and red, respectively. 

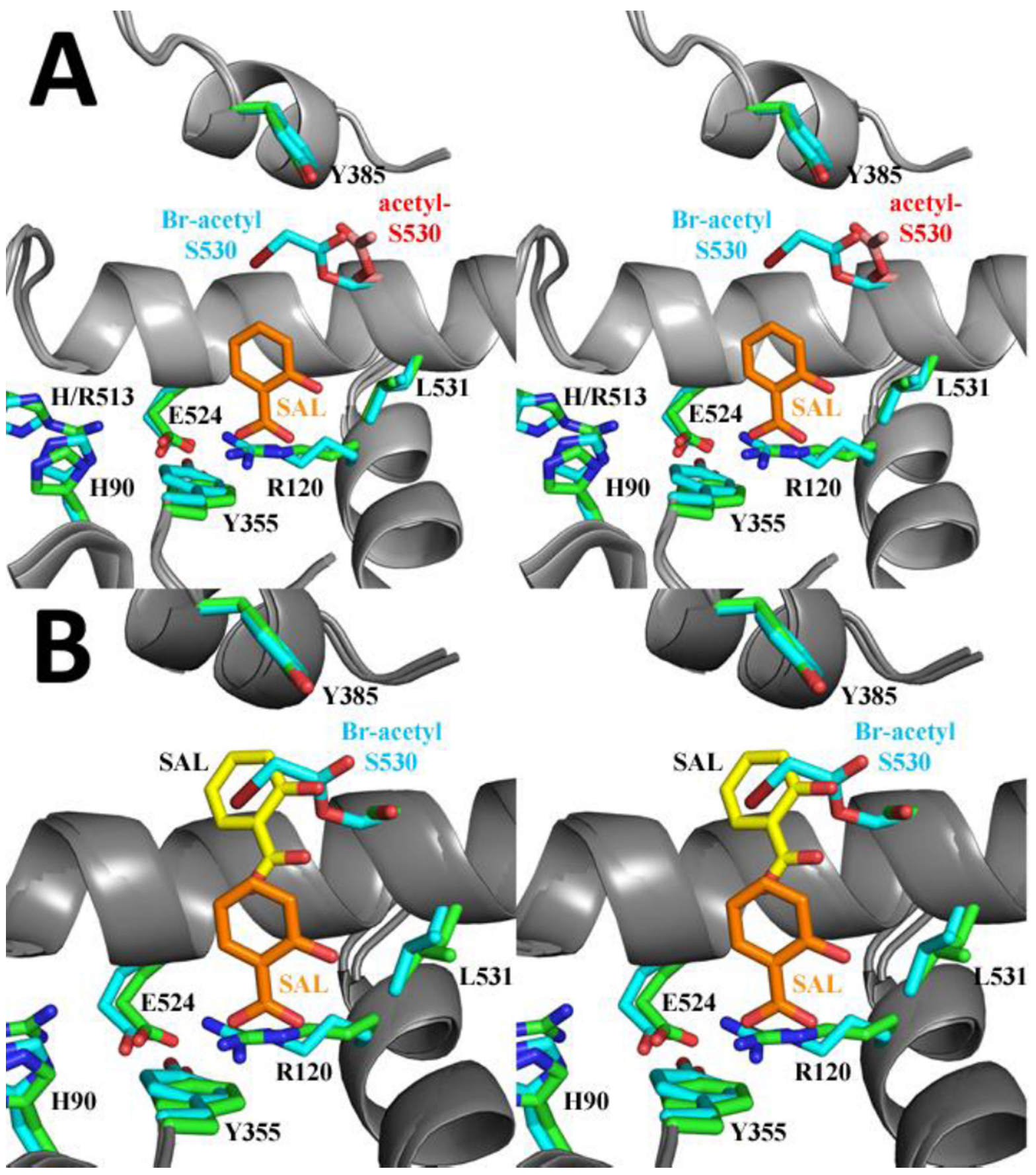

Figure 2. Comparison of Acetylated and Salicylate-bound huCOX-2 with Bromine-acetylated ovCOX-1

(A) Stereo view of the superposition of the acetylated Ser-530 side chain from monomer B of huCOX-2:ASA with the bromine-acetylated Ser-530 side chain from ovCOX-1 (PDB ID 1PTH; ${ }^{19}$ ). (B) Stereo view of the superposition of salicylate (yellow carbon atoms and red oxygen atoms) from monomer $\mathrm{B}$ of huCOX-2:SAL with salicylate bound in the cyclooxygenase channel of ovCOX-1 from 1PTH. The salicylate-leaving group from 1PTH is colored with orange carbon atoms and red oxygen atoms. Residues lining the 
cyclooxygenase channel of COX-2 are labeled accordingly, with carbon atoms colored green, while those from COX-1 are colored sky blue. Nitrogen and oxygen atoms in both structures are colored dark blue and red, respectively. 

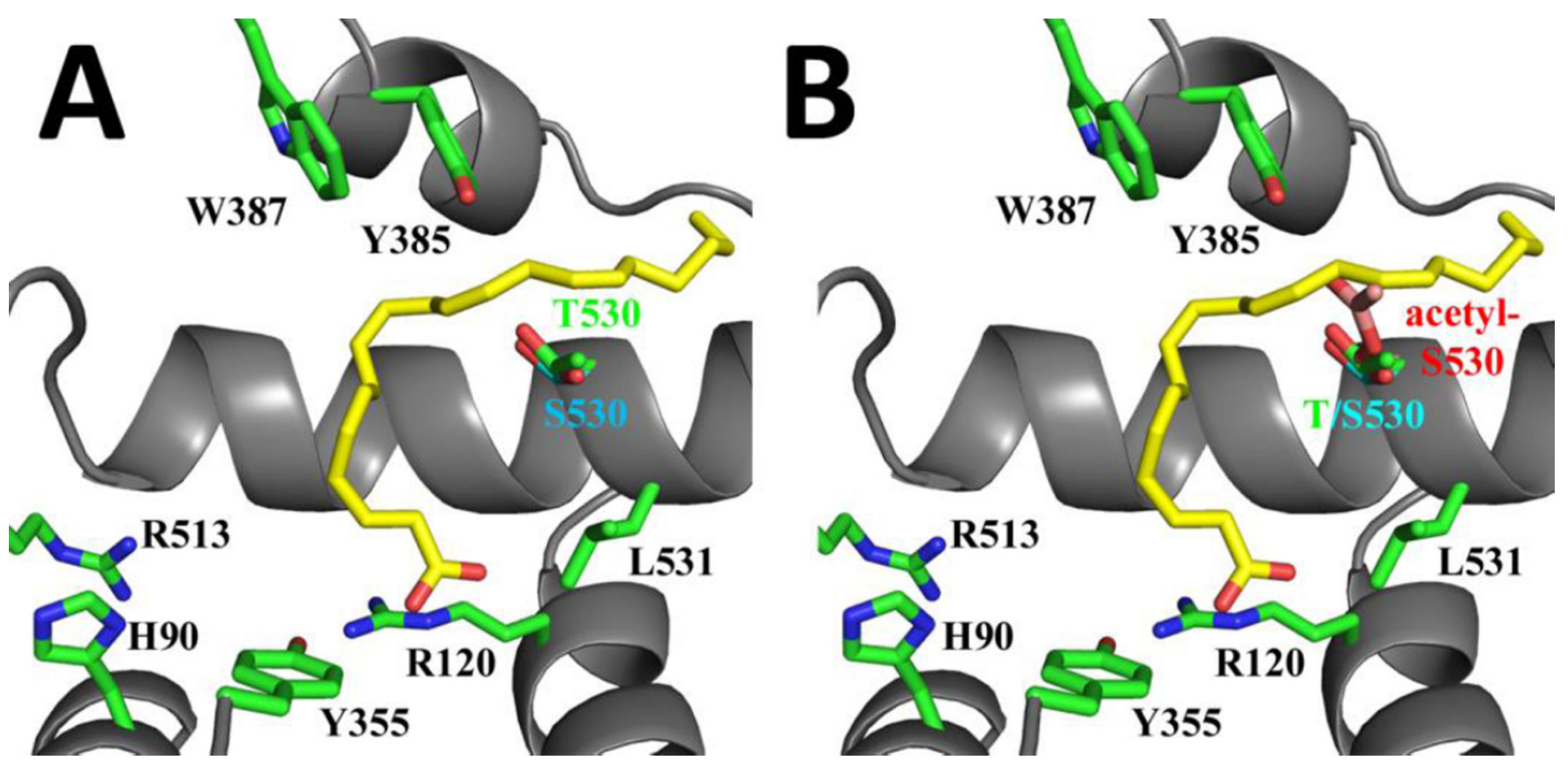

Figure 3. AA access to the hydrophobic groove in S530T muCOX-2

(A) Schematic of the cyclooxygenase channel from the S530T muCOX-2 crystal structure superimposed onto the wild type muCOX-2 crystal structure complexed with AA (pdb 3 HS5; ${ }^{7}$ ). The $\omega$-end of AA (yellow) would have full access to the hydrophobic groove in the S530T mutant. (B) Superposition of the aspirin-acetylated huCOX-2 crystal structure onto the view in A. Residues lining the cyclooxygenase channel are labeled accordingly, with carbon, nitrogen, and oxygen atoms colored green, blue, and red, respectively. 

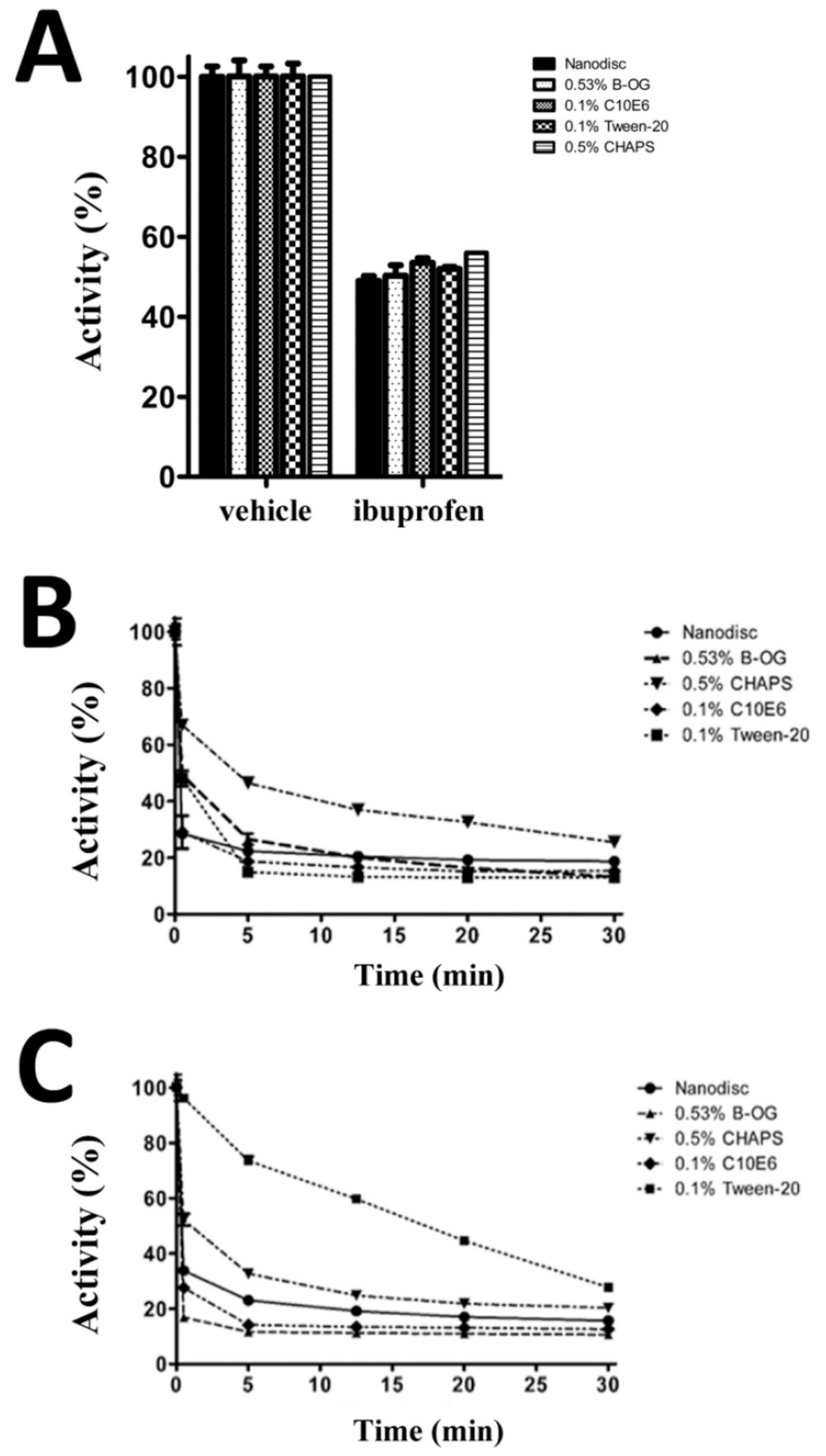

Figure 4. Inhibition of huCOX-2 in nanodiscs and various detergent systems Inhibition assays were performed as detailed in Experimental Methods to assess timedependent and time-independent inhibition of huCOX-2 in CHAPS, Tween-20, $\mathrm{C}_{10} \mathrm{E}_{6}, \beta O G$, and nanodiscs. (A) Time-independent inhibition was assessed using (R/S)-IBP. To assess time-dependent inhibition, a time course was performed and residual cyclooxygenase activity was measured at defined time points of $0.5,5,12.5,20$, and $30 \mathrm{~min}$ using (B) (R/S)FBP and (C) and celecoxib (CBX). 

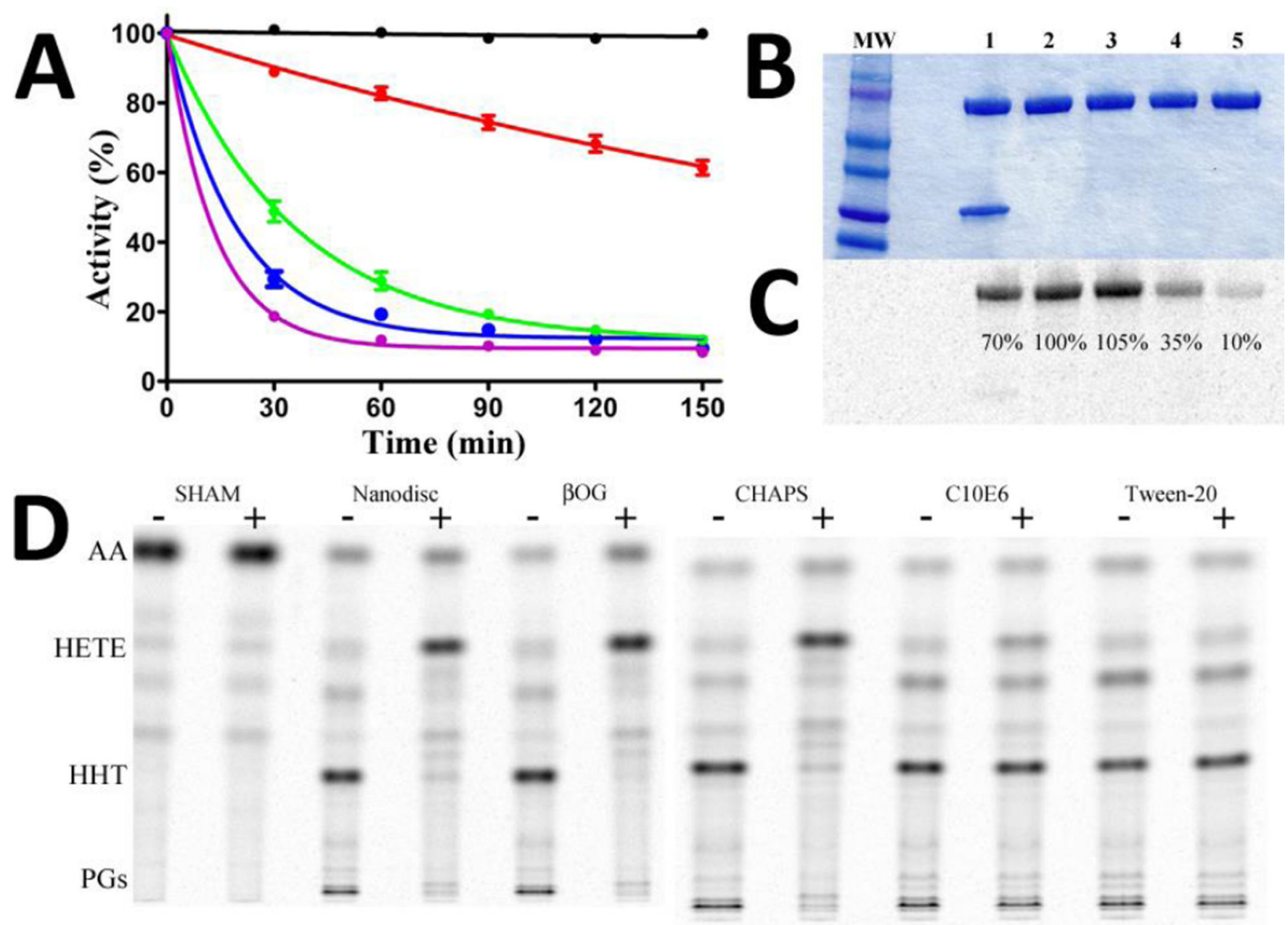

Figure 5. Differential acetylation of huCOX-2 in nanodiscs and various detergent systems (A) Aspirin inhibition of huCOX-2 in CHAPS (purple), Tween-20 (black), $\mathrm{C}_{10} \mathrm{E}_{6}$ (red), $\beta O G$ (green), and nanodiscs (blue) measured over time using an oxygen electrode. Values represent the average of triplicate measurements \pm S.E.M. (B) SDS-PAGE and (C) radiographic analysis of the acetylation of huCOX-2 by [acetyl- ${ }^{14} \mathrm{C}$ ] ASA. The percentages represent the intensity of the radioactive band relative to huCOX-2 prepared in $\beta O \mathrm{OG}$. Lane 1 , nanodisc-reconstituted huCOX-2; Lane 2, huCOX-2 in $\beta O G$; Lane 3, huCOX-2 in CHAPS; Lane 4, huCOX-2 in $\mathrm{C}_{10} \mathrm{E}_{6}$; Lane 5, huCOX-2 in Tween-20. Note, the lower molecular weight band in Lane 1 corresponds to the MSP of the nanodisc. MW, molecular weight marker. (D) TLC analysis of the products generated from $\left[1-{ }^{14} \mathrm{C}\right] \mathrm{AA}$ by huCOX-2 in the absence (-) or presence (+) of aspirin. PGs, prostaglandin products; HHT, 12hydroxyheptadecatrienoic acid; HETE, 11- and 15-hydroxyeicosatetraenoic acids. HHT is produced via nonenzymatic decomposition of PGs. 

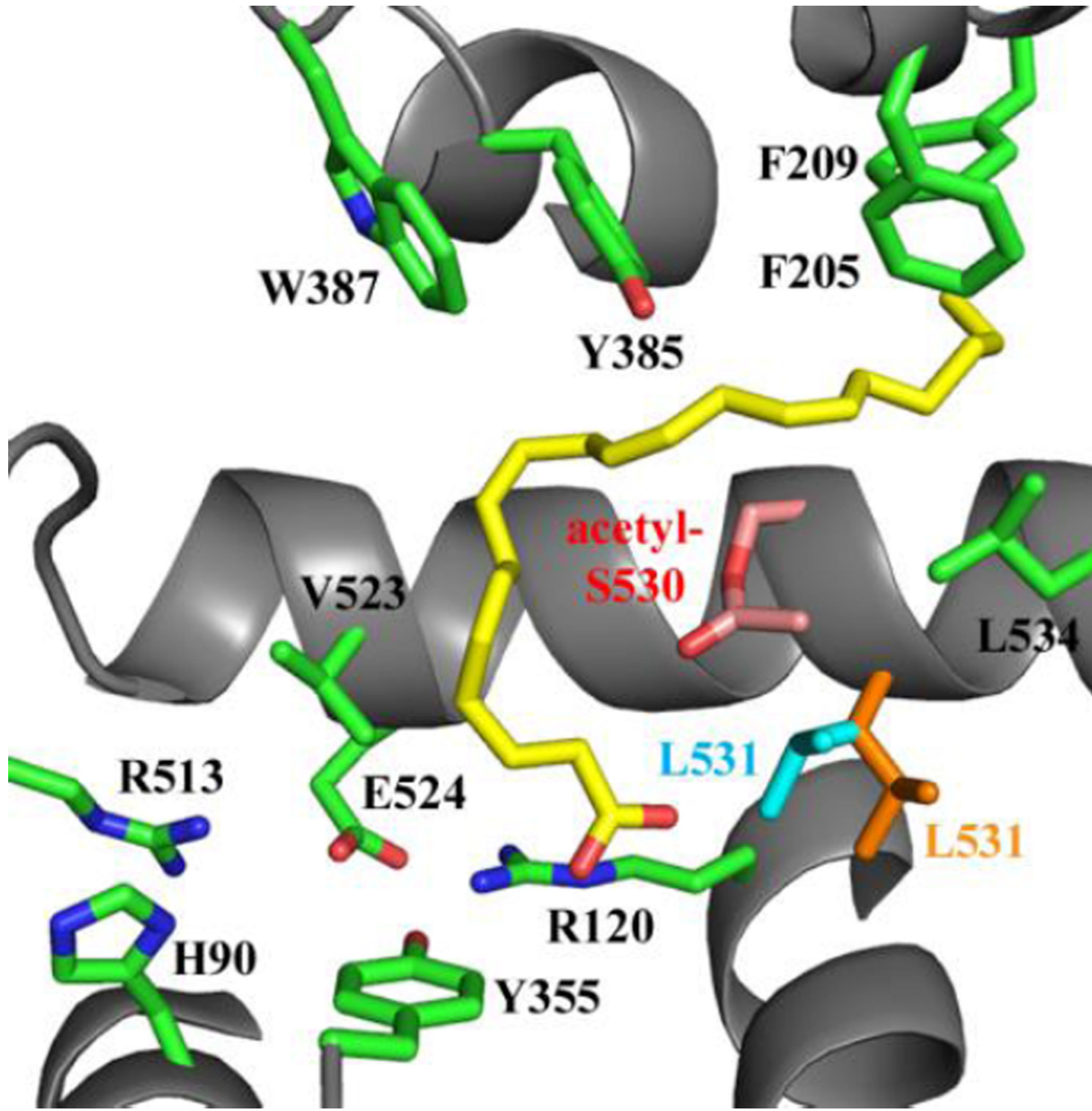

Figure 6. Model for 15R-HETE generation by acetylated huCOX-2

A model of AA (yellow) bound within the cyclooxygenase channel of aspirin-acetylated huCOX-2. The acetylated Ser-530 side chain (pink carbon atoms and red oxygen atoms) is proposed to adopt an alternate conformation, subsequently providing the $\omega$-end of AA access to the hydrophobic groove to achieve a productive binding pose leading to the generation of 15R-HETE. In conjunction, the side chain of Leu-531 (blue) would also adopt an alternate conformation (orange) to accommodate the moving acetylated side chain and the binding of AA within the cyclooxygenase channel. Residues lining the cyclooxygenase 
channel are labeled accordingly, with carbon, nitrogen, and oxygen atoms colored green, blue, and red, respectively. 
Table 1

Crystallographic statistics.

\begin{tabular}{|c|c|c|c|}
\hline $\begin{array}{l}\text { Crystallographic } \\
\text { Parameter }\end{array}$ & $\begin{array}{r}\text { huCOX2 } \\
\text { ASA }\end{array}$ & $\begin{array}{r}\text { huCOX2 } \\
\text { SAL }\end{array}$ & $\begin{array}{r}\text { muCOX2 } \\
\text { S530T }\end{array}$ \\
\hline Space group & $\mathrm{I} 222$ & $\mathrm{I} 222$ & $\mathrm{I} 222$ \\
\hline Number in asymmetric unit & 2 & 2 & 2 \\
\hline Wavelength $(\AA)$ & 1.033 & 1.033 & 0.978 \\
\hline \multicolumn{4}{|l|}{ Unit cell length $(\AA)$} \\
\hline $\mathrm{a}$ & 114.20 & 118.41 & 120.16 \\
\hline $\mathrm{b}$ & 130.13 & 132.66 & 132.50 \\
\hline $\mathrm{c}$ & 178.03 & 178.74 & 180.49 \\
\hline$a, \beta, \gamma\left(^{\circ}\right)$ & 90 & 90 & 90 \\
\hline Resolution $(\AA)$ & $53.88-2.04$ & $33.32-2.38$ & $20.0-1.90$ \\
\hline Highest Resolution Shell $(\AA)^{a}$ & $2.11-2.04$ & $2.42-2.38$ & $1.97-1.90$ \\
\hline Total observations & $269306(21791)$ & $241853(23169)$ & 436245 (32197) \\
\hline Total unique & 79455 (7361) & 59215 (5864) & $112453(10820)$ \\
\hline Multiplicity & $3.4(3.0)$ & $4.1(4.0)$ & $3.9(3.0)$ \\
\hline Completeness (\%) & $94.21(88.25)$ & $99.48(99.34)$ & $99.59(96.68)$ \\
\hline Mean I / $\sigma(\mathrm{I})$ & $8.99(2.36)$ & $8.99(2.15)$ & $10.57(1.88)$ \\
\hline $\mathrm{R}_{\text {merge }}(\%)^{b}$ & $9.0(46.6)$ & $11.2(70.2)$ & $8.6(58.3)$ \\
\hline $\mathrm{CC}^{1 / 2}$ & $0.995(0.698)$ & $0.994(0.698)$ & $0.992(0.712)$ \\
\hline $\mathrm{CC}^{* c}$ & $0.999(0.973)$ & $0.999(0.907)$ & $0.998(0.912)$ \\
\hline Wilson B-factor $\left(\AA^{2}\right)$ & 23.66 & 34.38 & 21.53 \\
\hline Number of atoms in refinement & 9982 & 9596 & 10382 \\
\hline $\mathrm{R}_{\text {work }}$ & $16.8(23.7)$ & $17.7(27.9)$ & $15.3(23.2)$ \\
\hline $\mathrm{R}_{\text {free }}{ }^{d}$ & $20.7(28.8)$ & $21.9(30.8)$ & $19.1(27.7)$ \\
\hline Average B-factor, protein $\left(\AA^{2}\right)$ & 29.9 & 43.6 & 24.0 \\
\hline Average B-factor, solvent $\left(\AA^{2}\right)$ & 38.0 & 41.8 & 35.9 \\
\hline Mean positional error $(\AA)^{e}$ & 0.228 & 0.295 & 0.186 \\
\hline RMSD bonds lengths $(\AA)$ & 0.003 & 0.006 & 0.012 \\
\hline RMSD bond angles $\left({ }^{\circ}\right)$ & 0.730 & 0.920 & 1.370 \\
\hline \multicolumn{4}{|l|}{ Ramachandran plot } \\
\hline Favored (\%) & 97 & 97 & 98 \\
\hline Allowed (\%) & 3 & 3 & 2 \\
\hline Disallowed (\%) & 0 & 0 & 0 \\
\hline Clash score $f$ & 2.35 & 3.16 & 2.69 \\
\hline
\end{tabular}

${ }^{a}$ Values in parentheses represent the values in the outermost resolution shell.

$b_{\text {Rerge }}$ as defined in 59.

${ }^{c} \mathrm{CC}^{1 / 2}$ and $\mathrm{CC}^{*}$ as defined in 60 . 


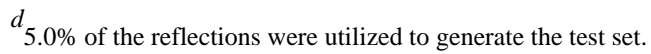

${ }^{e}$ Coordinate error as calculated by Luzzati plot.

$f_{\text {Clash score as calculated in MOLPROBITY }} 42$ 
Table 2

Kinetic characterization of huCOX-2 stabilized in nanodiscs and different detergent systems

$\mathrm{k}_{\mathrm{cat}}$ and $\mathrm{K}_{\mathrm{M}}$ values were derived from three independent determinations ( \pm S.E) using an oxygen electrode. AA was used as the substrate at concentrations between $2 \mu \mathrm{M}$ and $200 \mu \mathrm{M}$. Values for the relative peroxidase activity represent the average of two measurements, followed by normalization to the rate of the peroxidase activity of huCOX-2 in $\beta O G$.

\begin{tabular}{l|c|c|c|c}
\hline $\begin{array}{l}\text { Solubilization } \\
\text { System }\end{array}$ & $\begin{array}{c}\mathbf{k}_{\text {cat }} \\
\left(\mathbf{s}^{-\mathbf{1}}\right)\end{array}$ & $\begin{array}{c}\mathbf{K}_{\mathbf{M}} \\
(\boldsymbol{\mu M})\end{array}$ & $\begin{array}{c}\mathbf{k}_{\text {cat }} / \\
\mathbf{K}_{\mathbf{M}}\end{array}$ & $\begin{array}{c}\text { Rel. Peroxidase } \\
\text { Activity }\end{array}$ \\
\hline POPC-Nanodisc & 39.7 & $12.2 \pm 0.4$ & 3.3 & 95 \\
$\beta O G$ & 40.8 & $13.7 \pm 0.6$ & 3.0 & 100 \\
$\mathrm{C}_{10} \mathrm{E}_{6}$ & 49.9 & $10.7 \pm 0.5$ & 4.7 & 121 \\
Tween-20 & 49.3 & $10.0 \pm 0.5$ & 4.9 & 116 \\
CHAPS & 48.5 & $15.6 \pm 0.9$ & 3.1 & 105 \\
\hline
\end{tabular}

